\title{
MAINTENANCE DRUG THERAPY OF PANIC DISORDER
}

\author{
George C. Curtis, ${ }^{*}$ Juan Massana $\uparrow \uparrow$ Claudio Udina, $¥$ Jose Luis Ayuso $₫$ Giovanni B. \\ Cassanoß and Giulio Prrugi\$̧

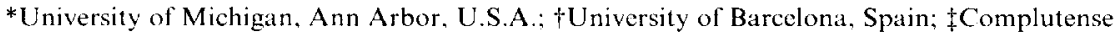 \\ University, Madrid, Spain and \$niversita Degli Studi di Pisa, Italy
}

\begin{abstract}
Summary-.. The efficacy of tricyclics and benzodiazepines in the short term (approximately 24 months) treatment of panic disorder is well demonstrated, but efficacy over the longer term is not considered established. The present study provided systematic data from a double blind comparison of maintenance therapy (up to 8 months) of panic disorder with or without agoraphobia with alprazolam, imipramine, or placebo in 181 patients who had responded to the same regimen in a randomized 8 -week treatment trial. All three groups had improved during the first 2 months (active treatments more than placebo and about equal to each other), and all maintained or extended their improvement over the next 6 months without any significant change in dose. More than twice as many alprazolam and imipramine than placebo patients (15\%) remained in treatment for the full 8 months and did slightly better on symptom measures than the remaining placebo patients. Both medications were well tolerated during the maintenance period. The data suggest sustained efficacy and safety of imipramine and alprazolam over an extended period. More specifically, they suggest that tolerance does not develop to the therapeutic effects of either drug.
\end{abstract}

\section{Introduction}

TRICYCLIC "antidepressants" (Ballenger, 1986; Liebowitz, 1985) and benzodiazepines (Ballenger, 1990; Ballenger et al., 1988; Cross-National Collaborative Panic Study Second Phase Investigators, 1992) have been found to be safe and effective in the acute treatment of panic disorder; i.e., up to about 8 weeks. The most extensively studied compounds are the tricyclic imipramine and the benzodiazepine alprazolam. For initiation of therapy, most of the advantage appears to lie with alprazolam, since its onset of therapeutic action is more rapid, and initial side effects are fewer and less troublesome. Within a month or less, however, the therapeutic effects of imipramine catches up, so that effectiveness is then about equal. Tricyclic side effects also appear to abate somewhat with time. Beyond the acute phase of drug treatment, i.e., the first month or two, there are many questions and little systematic data.

Questions concerning therapy over the longer term concern four main issues. The first concerns the natural history of the illness, and whether maintenance therapy is indicated at all. It does appear that in at least some cases the illness is chronic, and that in such cases maintenance therapy may be indicated (Noyes et al., 1990; Pollack et al., 1990). The second issue concerns withdrawal or rebound symptoms when drug treatment is stopped. It is now

This work was supported by the Upjohn Company.

*Author to whom correspondenec should be addressed at: University of Michigan, Department of Psychiatry, Med Inn Bldg 444, 1500 East Medical Center Drive, Ann Arbor, MI 48109-0840. U.S.A. 
clear that some such effects attend discontinuation of either class of compounds, but appear to be more troublesome in the case of benzodiazepines (Pecknold, Swinson, Kuch, \& Lewis, 1988; Rosenberg, 1990). It is not clear whether severity of drug discontinuation effects are related to duration of therapy. The third issue concerns whether additional side effects occur during maintenance therapy, and the fourth is whether therapeutic effectiveness is stable over time, or more specifically, whether tolerance develops to the therapeutic effects. This report deals with the latter two issues; i.e., efficacy and side effects over the longer term in a large multicenter trial of imipramine, alprazolam, and placebo. Data from that trial pertaining to short term effects (Cross-National Collaborative Panic Study Second Phase Investigators, 1992), drug discontinuation effects (Rosenberg, 1990), and relapse and long term outcome (Katschnig, 1990) are reported elsewhere.

Such documentation as is currently available on efficacy and side effects during maintenance therapy is mostly retrospective in nature, coming from follow-up of patients 1-6 years after initial treatment in acute study protocols, followed by naturalistic treatment until the time of follow-up (Aronson, 1989; Katschnig, 1990; Nagy, Krystal, Woods \& Charney, 1989; Noyes et al., 1988, 1990; Pollack, Tesar, Rosenbaum, \& Spier, 1986). At follow-up a high proportion of patients are usually found to have remained on medication, frequently at reduced dose, to have maintained or extended their improvement and to have tolerated continued medication therapy quite well for extended periods of time. In these studies, however, the effects of multiple treatments are difficult to separate, and systematic information on clinical condition and medication doses during the course of maintenance therapy is usually not available. In a study by Burrows (1990), patients with panic disorder on maintenance therapy with alprazolam were reported to tolerate the treatment quite well and to maintain their improvement without escalation of dose. The present study has several features apparently not reported elsewhere. Patients from an acute treatment study were maintained on the same double blind treatment with either alprazolam, imipramine or placebo for a total of 8 months, while all other treatments were excluded, and systematic data collection was carried out at monthly intervals.

Data for the study were obtained during the Cross-National Collaborative Panic Study, Phase II, sponsored by the Upjohn Company, Kalamazoo, Michigan. The Cross-National trial was conducted in two phases. In Phases I and II, alprazolam was superior to placebo in an 8-week acute treatment period (Ballenger et al., 1988; Cross-National Collaborative Panic Study Second Phase Investigators, 1992). Both drugs were well tolerated (Cassano, 1990; Noyes et al., 1988), but discontinuation of alprazolam was accompanied by a transient symptom rebound in $8-27 \%$ and withdrawal symptoms in approximately $35 \%$ of patients despite gradual reduction in dose (Pecknold et al., 1988; Rosenberg, 1990). Phase I patients whose treatment periods were extended from 24 to 52 weeks maintained their improvement, with a slight reduction (rather than an increase) in average dose (Burrows, 1990). In Phase II imipramine was also superior to placebo during the initial 8 weeks and approximately equal to alprazolam on most measures, but alprazolam had a more rapid onset of action, fewer side effects and fewer dropouts (Cross-National Collaborative Panic Study Second Phase Investigators, 1992).

Four of the 12 Phase II study sites participated in a study of maintenance drug therapy for 6 additional months following the initial 2-month acute treatment period. 


\section{Methods}

The design and methods of the overall Cross-National Collaborative Panic Study have been described in detail elsewhere (Klerman, 1988; Klerman, Coleman, \& Purpura, 1986). Briefly, there were two phases. Phase I was a multicenter randomized, double blind, flexible dose trial of alprazolam versus placebo in the treatment of panic disorder with or without agoraphobia, providing data from 481 patients (Ballenger et al., 1988). Phase II employed similar methods and design, but included an imipramine cell in addition to the alprazolam and placebo cells, and provided data on 1,010 patients in the acute treatment study.

Phase II was conducted at 12 sites in Western Europe, South America, and North America. Patients with a history of DSM-III major depression beginning prior to the onset of panic disorder were excluded. Diagnoses were arrived at by the Structured Clinical Interview for DSM-III (SCID), modified for this project (SCID-UP). A flexible dosing schedule was employed with adjustments according to tolerance and judged therapeutic need. Maximum dose was 10 tablets per day, representing $10 \mathrm{mg}$ of alprazolam or $225 \mathrm{mg}$ of imipramine. Change measures reported here include drug dose, retention in the study, total number of panic attacks per week, overall phobia score, Hamilton Anxiety Scale, and Physicians' global improvement ratings.

Patients at four selected study sites (Belgium, Brazil, Italy and Spain), who completed the acute phase were invited to continue the same double blind treatment for an additional 6 months if they were judged to have benefited from their treatment to that point. A total of 181 patients accepted the invitation and entered this phase of the study: 78 were on alprazolam, 65 on imipramine, and 38 on placebo, representing $58.2 \%, 47.1 \%$, and $27.3 \%$, respectively, of the patients entering the acute treatment study from those sites. Patients were seen monthly. Clinical assessments, ratings, and dose adjustments under double blind conditions were conducted as in the acute study.

Extensive testing had indicated good randomization of the initial 1,010 patients. Since patients entering the maintenance therapy trial were a selected subset of the initial randomized sample, additional tests were performed to evaluate the representativencss of the maintenance therapy sample with respect to the original randomized sample and the comparability of the three maintenance therapy groups to each other. Patients from the 4 sites who did and did not subsequently enter the maintenance treatment trial were compared at baseline prior to any treatment and again at the end of acute treatment (week 8). Then, patients in the alprazolam, imipramine and placebo groups who entered the maintenance therapy study were compared with each other at baseline prior to any treatment, and again at the end of acute treatment (week 8). Variables used for these comparisons included (where applicable) study site, treatment assignment, age, sex, panic disorder subtype (uncomplicated, limited avoidance, extensive avoidance), duration of illness, presence of current or past major depression, total panic attacks per week, anticipatory anxiety intensity, percentage of time spent in anticipatory anxiety, overall phobia severity, SCL-90 total score, Hamilton Anxiety, Hamilton Depression, ratings of work and social disability, medication dose, and physicians' global improvement ratings. Adverse effects were assessed using a modification of SAFTEE, which was developed at the NIMH Psychopharmacology Research Branch (Levine \& Schooler, 1986). Also, the physician prepared a descriptive report of any intercurrent illnesses or medical events which were considered clinically 
significant, whether or not they were thought to be drug related. These reports included the time of onset, actions taken if any, and a judgment of whether the event was drug related.

To compare across treatments during maintenance therapy, and across time within treatments, only descriptive statistics (central tendency and error of estimate) were performed on measures of change. This limitation was observed because the patients entering the maintenance treatment study were no longer strictly randomized groups, and thus did not satisfy conventional requirements for performing comparative statistics. On the other hand, the comparability of the different drug groups to each other and their representativeness of the total randomized samples on important clinical variables were known and did not appear to be significantly biased except with respect to the response to their assigned treatment. By inspection of graphic displays of means and $95 \%$ confidence intervals, it was possible to estimate which differences would have been significant if comparative statistics had been carried out.

\section{Results}

\section{Adequacy of randomization}

By far the largest differences between patients who did and did not enter the maintenance treatment study were the site of study and the treatment assignment, both being highly significant statistically. As is shown in Tables 1 and 2, more patients in Italy and Brazil and fewer in Belgium entered the extended study. Reasons for the site differences were not entirely clear, but they appeared to relate to different patient management styles and different practice conditions in the countries. Also, more patients assigned to alprazolam than to imipramine entered the maintenance therapy study, and far more assigned to either active treatment than to placebo entered.

Table I

Patient Enrollment by Country

\begin{tabular}{lccc}
\hline & $\begin{array}{c}\text { Acute } \\
\text { phase } \\
\text { ITT \# }\end{array}$ & $\begin{array}{c}\text { Chronic phase } \\
\text { \# entered }\end{array}$ & $\begin{array}{c}\% \text { of ITT } \\
\text { entered }\end{array}$ \\
\hline $\begin{array}{l}\text { Belgium } \\
\text { Brazil }\end{array}$ & 99 & 16 & $16.2 \%$ \\
Italy & 106 & 58 & $54.7 \%$ \\
Spain & 100 & 61 & $61.0 \%$ \\
Total & 106 & 46 & $43.4 \%$ \\
& 411 & 181 & $44.0 \%$ \\
\hline
\end{tabular}

Note. ITT $=$ intent-to-treat.

Additional baseline predictors of progression to the maintenance therapy study were anticipatory anxiety intensity, which was slightly but significantly higher in those who entered, and Hamilton Anxiety and Hamilton Depression scores which were slightly but significantly higher in those who did not enter. The entry and non-entry groups did not differ significantly at baseline on any of the other comparison variables.

As intended, at the end of the acute treatment (week 8), patients who entered the maintenance therapy study were in better clinical condition than those who did not, as 
Table 2

Patient Enrollment by Treatment

\begin{tabular}{lccc}
\hline & $\begin{array}{c}\text { Acute } \\
\text { phase } \\
\text { \# ITT }\end{array}$ & $\begin{array}{c}\text { Chronic phase } \\
\text { \# entered }\end{array}$ & $\begin{array}{c}\% \text { of ITT } \\
\text { cntercd }\end{array}$ \\
Alprazolam & 134 & 78 & $58.2 \%$ \\
Imipramine & 138 & 65 & $47.1 \%$ \\
Placebo & 139 & 38 & $27.3 \%$ \\
Total & 411 & 181 & $44.0 \%$ \\
& Chi-square $=27.2 p=.000$ & \\
\hline
\end{tabular}

Note. ITT $=$ intent-to-treat.

indicated by fewer total panic attacks per week, and by lower scores on overall phobia, anticipatory anxiety intensity, total SCL-90, Hamilton Anxiety, Hamilton Depression, and work and social disability, and physicians' ratings of global improvement. All differences were highly significant statistically, $p$ values ranging from $<.01$ to $<.0001$. No other differences between the groups were significant at this point in time.

At pre-acute phase baseline, only one marginally significant $(p=.053)$ difference was detected among the alprazolam, imipramine and placebo groups entering the maintenance study. This was in the mean total SCL-90 score, the placebo group being slightly lower than the active treatment groups.

At the end of the acute treatment period (week 8), the active treatment groups entering the maintenance therapy study were significantly better than the placebo group with respect to anticipatory anxiety intensity, overall phobia, Hamilton Anxiety, and physicians' global improvement ratings. Since none of these differences were present prior to treatment, the findings suggest that, although only patients who had improved were invited to continue, the patients on active treatments had improved somewhat more than those on placebo.

\section{Dropouts before and during maintenance therapy}

Figure 1 graphs the percentage of patients, with $95 \%$ confidence intervals, from the 4 study sites combined, who remained in the study at each time point from the acute phase baseline through the final maintenance therapy evaluation at month 8 of the study. Points where $95 \%$ confidence intervals do not overlap represent statistically significant differences. At month 2, the end of the acute treatment study, the alprazolam group had a significantly higher retention rate $(84 \%)$ than either the imipramine $(68 \%)$ or placebo groups $(62 \%)$. From that point on, the difference between alprazolam and imipramine narrowed and, at the end, essentially identical percentages of patients in the alprazolam group (37\% of those randomized, $61 \%$ of those entering maintenance) and in the imipramine group (35\% of those randomized, $74 \%$ of those entering maintenance) remained in the study. Both active treatment groups retained significantly more patients throughout the maintenance therapy study than did the placebo group, in which only $15 \%$ of the initial randomized sample ( $55 \%$ of those entering maintenance) completed the maintenance therapy study. 


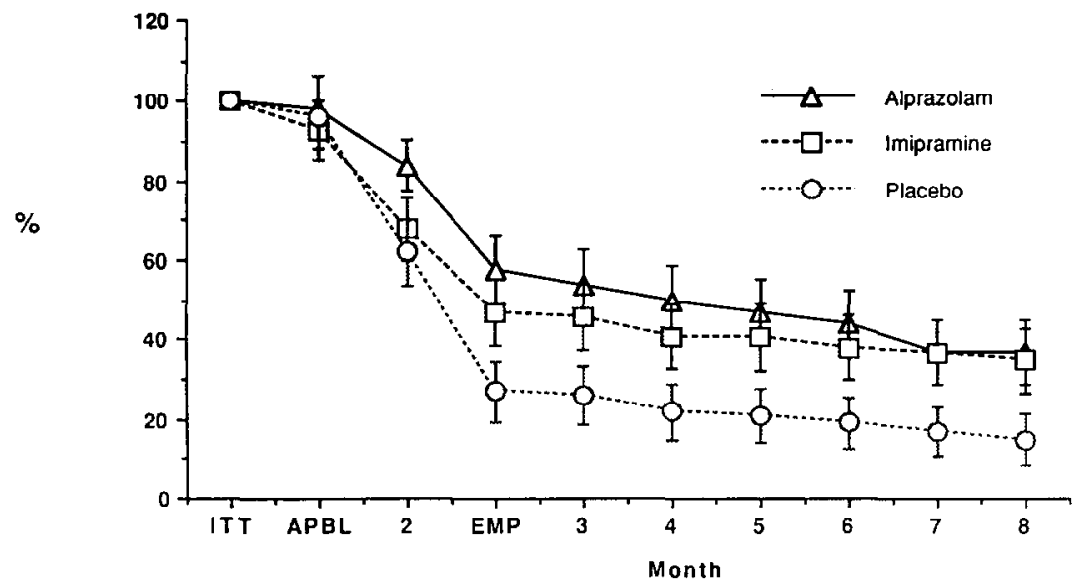

Figure 1 . Retention in study with $95 \%$ confidence levels. ITT $=$ intent-to-treat. APBL $=$ acute phase baseline. Month 2 = end of acute phase. EMP = enter maintenance phase.

\section{Stability of dose}

At each visit the clinician had the option of adjusting the medication dose if the clinical condition of the patient so indicated. Figure 2 graphs the average number of tablets taken per day from beginning to end of the maintenance therapy study. The doses were essentially identical and unchanging at between 6 and 7 tablets per day, representing either that number of placebo tablets, or 6 or $7 \mathrm{mg}$ of alprazolam, or 150 or $175 \mathrm{mg}$ of imipramine per day. If comparative statisties had been performed, there would have been no significant differences between medication groups at any time point and no significant changes with time in any medication group.

\section{Efficacy measures during maintenance therapy}

Figure 3 graphs total number of panic attacks per week with $95 \%$ confidence intervals from the acute study baseline to the end of the maintenance therapy study. Plots for the groups are presented separately rather than superimposed because some of the information is obscured in the superimposed plots. It appears that if comparative statistics had been performed, there would have been no significant differences between treatment groups from beginning to end of the maintenance therapy study. However, the very wide confidence intervals in the placebo group indicates that some patients continued to have significant numbers of panic attacks, whereas virtually no attacks occurred from month 4 onward among patients remaining in the imipramine group, as indicated by near-zero means and near-zero $95 \%$ confidence intervals. Patients in the alprazolam group continued to have infrequent attacks. The endpoint values are the mean and confidence intervals of the last value obtained for all subjects in the maintenance therapy study, regardless of whether they dropped out prior to the end or remained until the end. The relatively large differences in mean and confidence interval between month 8 and the endpoint in the placebo group 


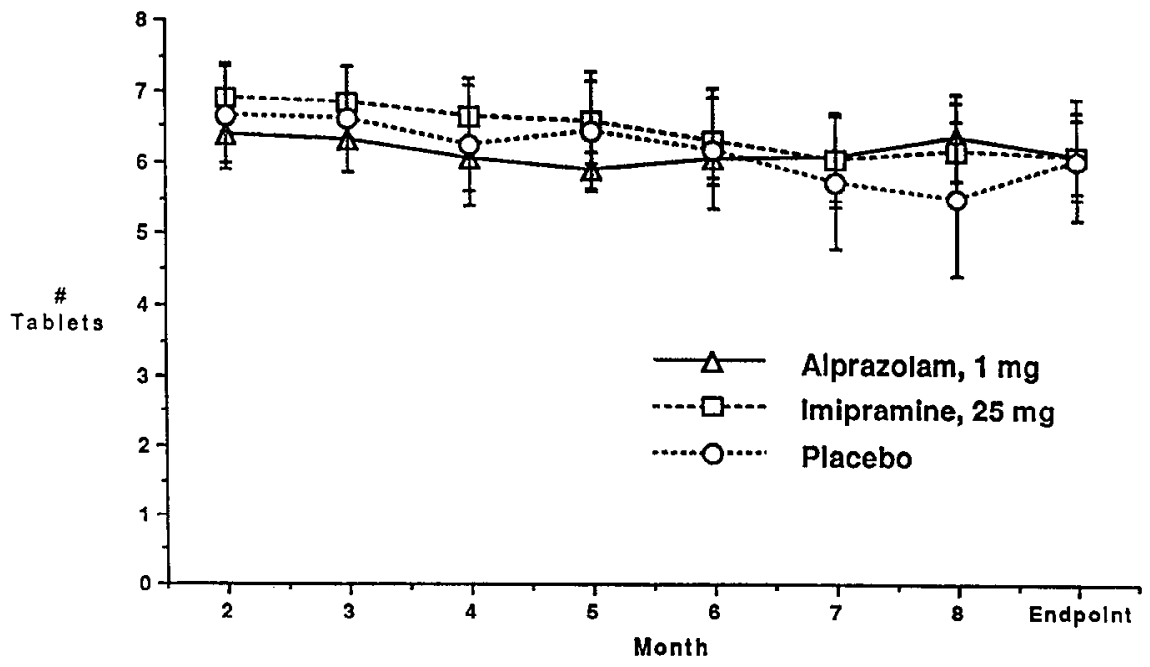

Figure 2. Average number of tablets per day with $95 \%$ confidence intervals.

suggests a trend for patients who dropped out to have been having more panic attacks at the time of dropout than completers were having at the time of completion.

Figure 4 graphs overall global phobia scores. Comparative statistics would probably have indicated no significant differences between groups or within groups over time. However, the somewhat higher means and wider confidence intervals in the placebo group suggest that some placebo patients did relatively poorly on this measure compared to the other groups. The comparatively large difference between month 8 and endpoint for the placebo group again shows that placebo dropouts tended to be more symptomatic at the time of dropout than were completers at the time of completion.

Figure 5 graphs means and 95\% confidence intervals of Hamilton Anxiety Scale scores. Comparative statistics would probably have indicated few or no differences between treatments at any time point. However, the early and late confidence intervals of imipramine subjects sometimes fail to overlap, suggesting the possibility of a slight continuing improvement from early to late maintenance therany. Again, the placebo group showed a somewhat greater difference between endpoint and completer values.

Figure 6 graphs means and 95\% confidence intervals of physicians' global improvement scores. Placebo patients did somewhat less well throughout the maintenance therapy study, but only occasionally did the $95 \%$ confidence intervals fail to overlap those of the active treatment groups. Alprazolam and imipramine confidence intervals usually overlapped. Over time the alprazolam group remained stable, but in the imipramine group, early and late confidence intervals sometimes failed to overlap, suggesting possible slight continuing improvement in the imipranine group. Some apparent improvement may have been due to dropping out by patients who were doing less well. This effect appears to have been smaller in the active treatment groups than in the placebo group, judging by the difference between week 8 and endpoint scores. 

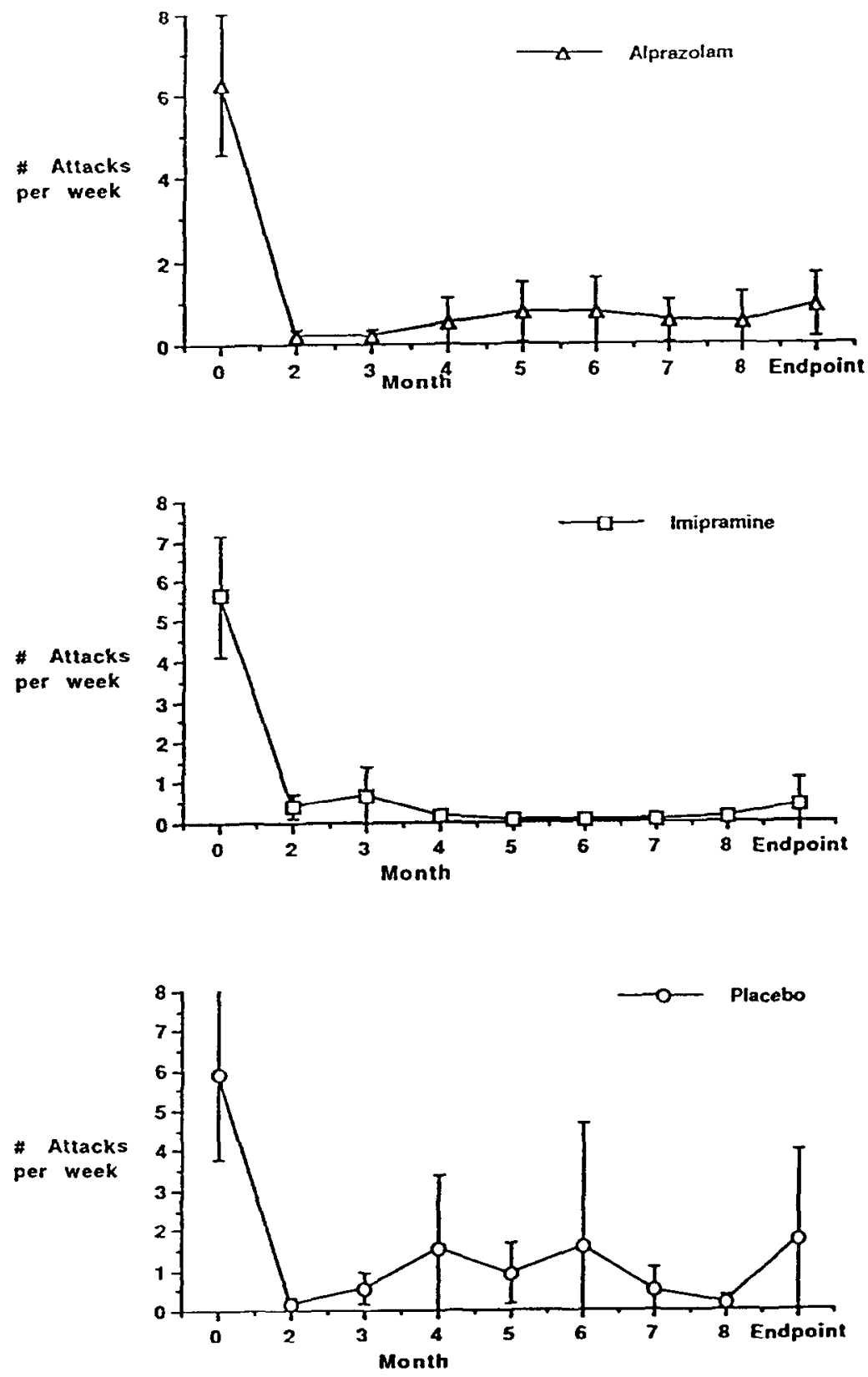

Figure 3. Total panic attacks/weck with $95 \%$ confidence levels. 

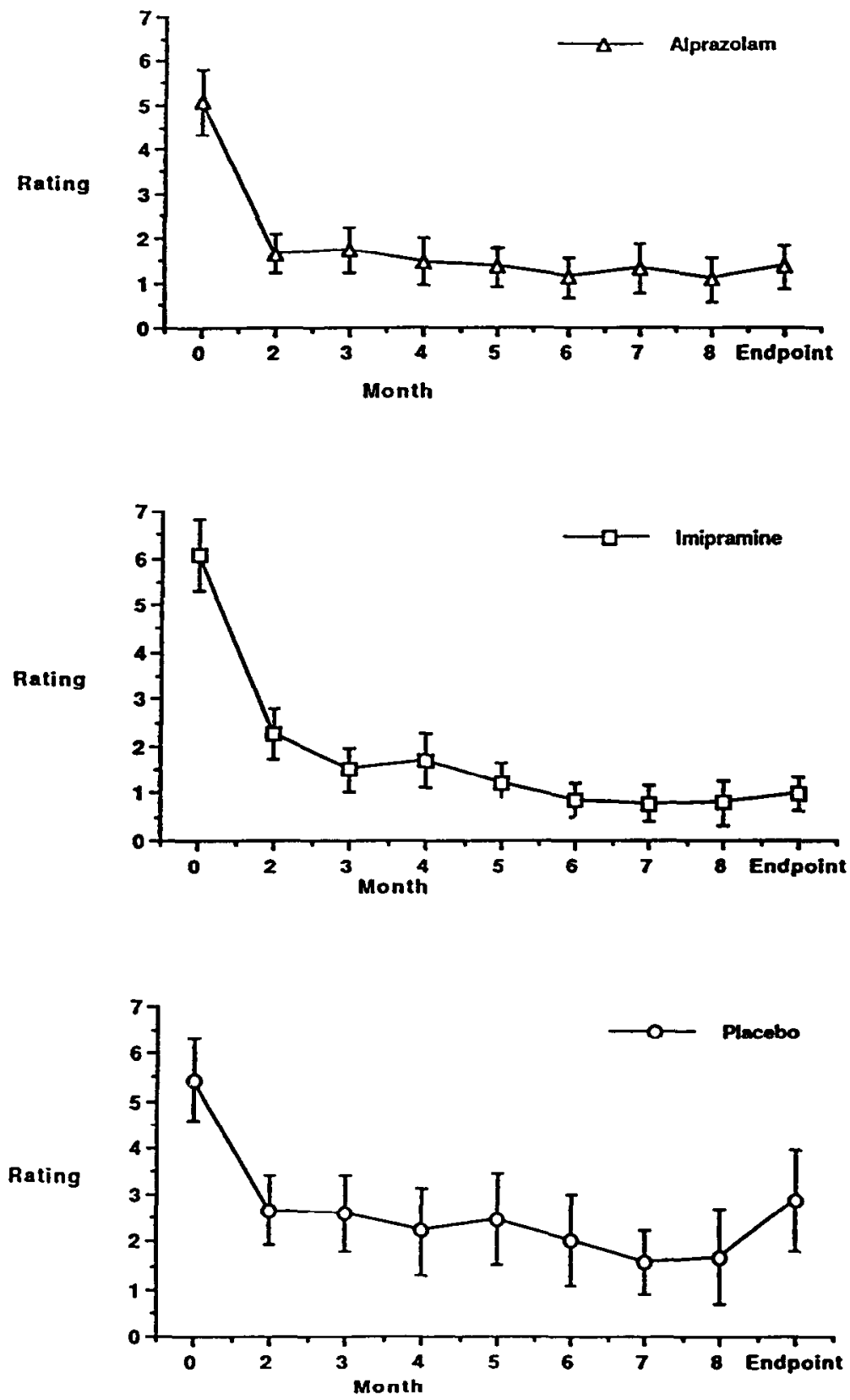

Figure 4. Overall global phobia ratings with $95 \%$ confidence intervals. 

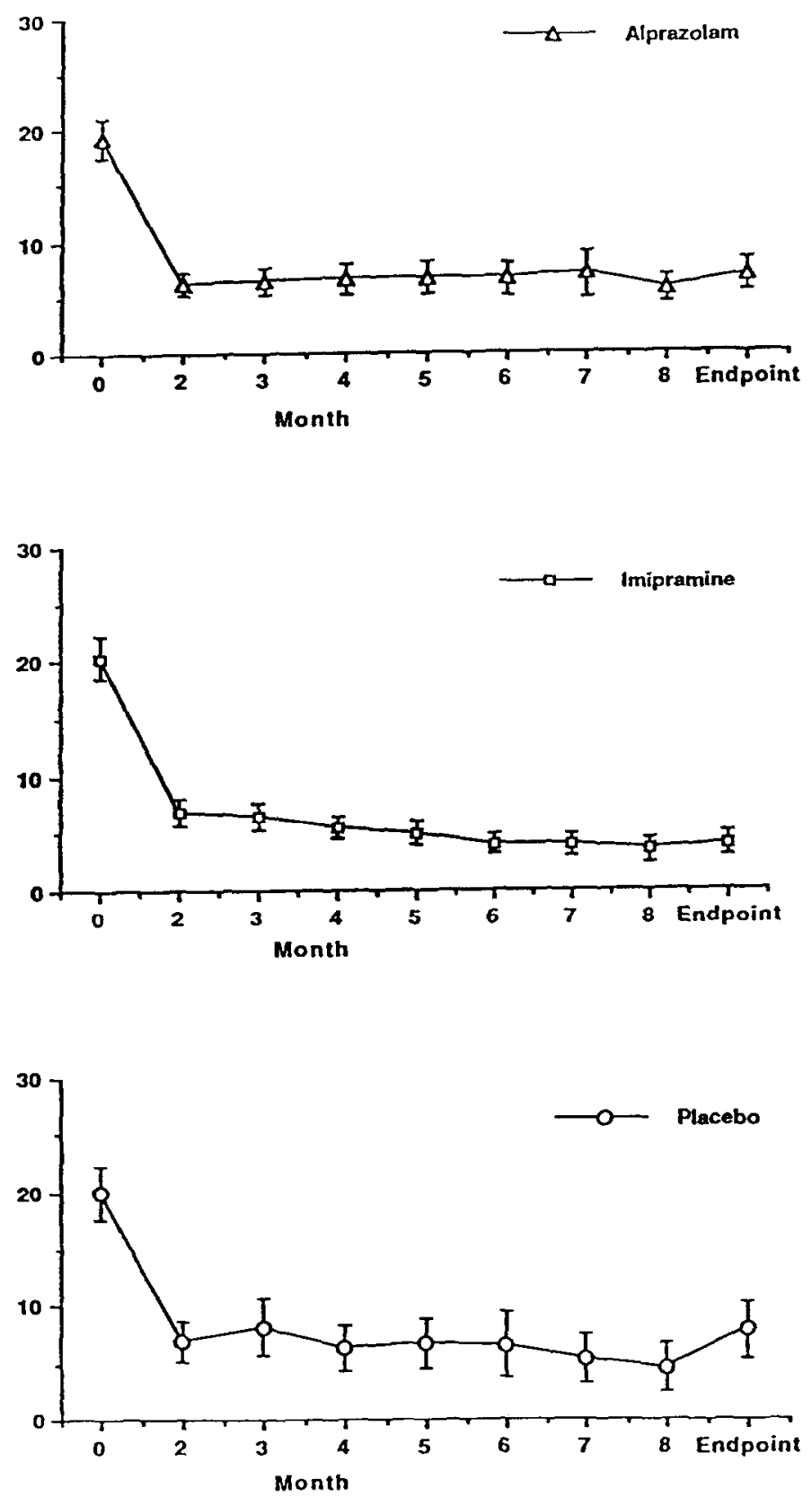

Figure 5. Hamilton Anxiety Scale with $95 \%$ confidence intervals. 

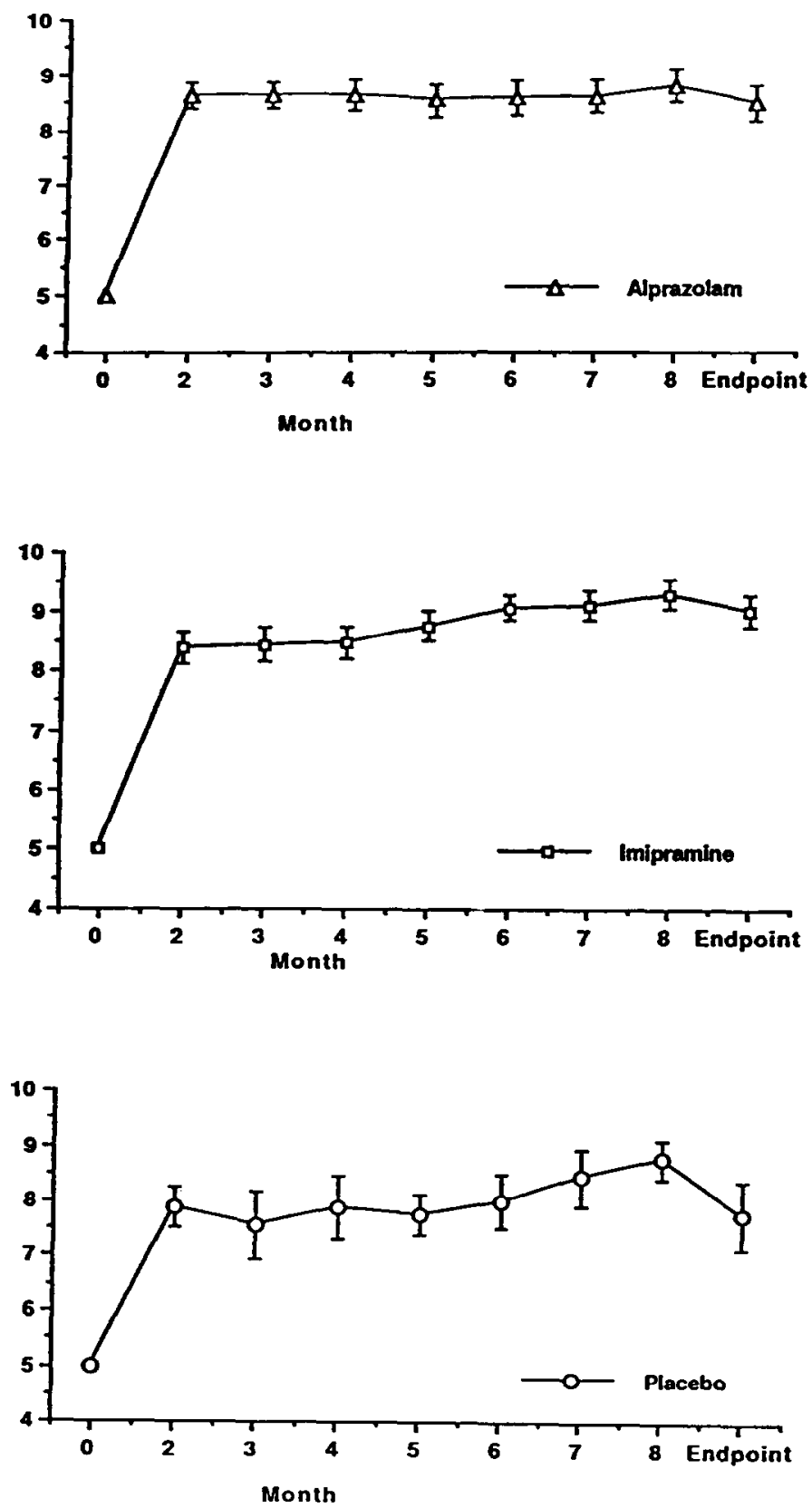

Figure 6. Physicians' global improvement with $95 \%$ confidence levels. 


\section{Unwanted effects during maintenance therapy}

Side effects were minor and infrequent in all groups. Table 3 lists the percentage of patients in whom the most frequent unwanted effects were found in at least one visit during the maintenance therapy study. In order to provide documentation without excessive minor detail, Table 3 includes only those symptoms which were found at least once at an intensity higher than at short term baseline in at least $10 \%$ of the patients in an active treatment group, provided that the frequency of that finding was higher in that active treatment group than in the placebo group. Notably, $83 \%$ of placebo patients had at least one such symptom on at least one visit. This exceeded the frequency for both the alprazolam $(80 \%)$ and the imipramine $(74 \%)$ groups.

Table 3

Percent of Patients Experiencing Various Unwanted Effects at an Intensity Greater than Baseline on at Least One Visit During Maintenance Therapy

\begin{tabular}{lccc}
\hline Symptom & Alprazolam & Imipramine & Placebo \\
\hline Abnormal diastolic blood pressure & 23 & 13 & 22 \\
Abnormal weight change & 22 & 36 & 14 \\
Weight loss & 21 & 6 & 6 \\
Increase in appetite & 4 & 19 & 12 \\
Insomnia & 12 & 3 & 6 \\
Malaise & 10 & 5 & 6 \\
Irritability & 15 & 3 & 3 \\
Dry Mouth & 12 & 2 & 3 \\
Tremor & 10 & 5 & 0 \\
Nausea/vomiting & 11 & 12 & 9 \\
Constipation & 20 & 74 & 3 \\
Any side effect & 80 & 14 & 83 \\
Any medical event & 27 & & 18 \\
\hline
\end{tabular}

Note. Items from a list of 46 are included if the symptom occurred in at least $10 \%$ of patients on either active drug and if that figure also exceeded the percentage for placebo. Abnormal diastolic pressure defined as $\geq 90 \mathrm{~mm} \mathrm{Hg}$. Abmormal weight change defined as $\geq 5 \%$ change from short term baseline. Medical events included pregnancy, transient fevers, psychiatric symptoms, intercurrent illnesses, etc.

In the imipramine group the frequency of most side effects was less than in either the placebo or the alprazolam group, with the exception of increased appetite (19\% of patients), and abnormal weight change $(36 \%)$. Even constipation, which is a recognized side effect of imipramine was reported less $(12 \%)$ than in the alprazolam group $(20 \%)$ though more than in the placebo group (3\%). Dry mouth, perhaps the most common early side effect of imipramine therapy, was not among the more frequently reported side effects, being reported at least once during the maintenance therapy study by $12 \%$ of alprazolam patients and only $3 \%$ of imipramine and placebo patients. The following symptoms were found as described in $10 \%$ or more of one or the other treatment groups but were onitled from reporting because they were found in a higher percentage of placebo patients: dizziness/faintness, headache, stomach/abdominal discomfort, decrease in appetite, and weight gain. 


\section{Discussion}

These results clearly indicate that initial therapeutic gains with imipramine or alprazolam are sustainable by maintenance therapy for periods up to at least 8 months, without the need for dose escalation, and without serious development of side effects. This is consistent with conclusions based on retrospective naturalistic follow-up studies ( $\Lambda$ ronson, 1989; Katschnig, 1990; Nagy et al., 1989, 1990; Noyes, Garvey, Cook, \& Samuelson, 1989; Pollack et al., 1986) and with data collected during maintenance therapy with alprazolam or placebo from a subset of patients from Phase I of the Cross-National Collaborative Study (Burrows, 1990). The problem is, that from the present study the same could be said of placebo treatment. However, because of the pattern of dropouts, things are not quite that simple.

Unfortunately, reasons for dropouts were not recorded during the maintenance study. In the immediately preceding acute treatment study the most common reasons given for dropouts were ineffectiveness of medication, side effects, and patients refusing treatment for reasons not specified (Cross-National Collaborative Panic Study Second Phase Investigators, 1992). In that study and in the present one, mean endpoint values (last value obtained from every patient) were rather consistently worse than mean completer values (last value of all patients completing the study), suggesting that dropouts were doing less well than completers. All of this is consistent with the common sense inference that not doing well may be a major reason for dropping out. To the extent that this is the case, dropouts will inflate the apparent efficacy of all treatments, both active and inactive. Also, to the extent that this is true, more dropouts mean more inflation. In this study, the retention rate of the placebo group was only about half that of the active treatment groups, suggesting that apparent placebo improvement was more inflated than apparent improvement on alprazolam or imipramine. Even so, the remaining alprazolam and imipramine patients did slightly better than placebo patients on most measures, though only occasionally the $95 \%$ confidence intervals failed to overlap. Thus it is at best dubious to conclude that placebo was actually as effective for maintenance therapy as alprazolam or imipramine.

It is much harder to identify any advantage of one of the active drugs over the other. For the first 2-4 weeks of treatment, alprazolam appeared to be more effective and have fewer side effects than imipramine (Cross-National Collaborative Panic Study Second Phase Investigators, 1992; Ballenger et al., 1988). Thereafter, the advantage narrowed, and in the present study, appeared to be completely gone by 8 months. In fact there were slight hints of a trend favoring imipramine during the final months of the maintenance study.

Another limitation of the study is that the groups entering the maintenance therapy study were no longer randomized, but emerged by self- and clinician-selection from groups which had been randomized for the purpose of the acute treatment study. Selection was primarily based on improvement and tolerance for their assigned treatments, though unidentified factors operating locally at the study sites also clearly played a large role. Nevertheless the selection process did not appear to bias or unbalance the treatment samples in any obvious or very significant way detectable by conventional tests for adequacy of randomization.

The findings in the imipramine group are consistent with other reports (Aronson, 1989; Noyes et al., 1989), suggesting that around $35-50 \%$ drop out early, primarily because of side effects, but that those who remain improve and maintain their improvement for periods of months to years. However, Aronson's patients received behavior therapy at the beginning, 
and this could have had some part in maintenance of improvement. This was not a factor in the present study, since all formal psychotherapies were excluded while drug treatment was in progress. Apparently the main problem with imipramine early in treatment is overstimulation, and the main problem later is weight gain. Some of the early troublesome anticholinergic side effects of imipramine, specifically dry mouth and constipation, appear to wane with prolonged treatment, either as a result of tolerance or of dropout.

For the design of future studies the main points of improvement would be preserving the randomization, and reducing the altrition and keeping careful account of reasons for dropouts. Trials over longer periods of time with long follow-up intervals are also desirable but difficult to implement. Part of preserving the randomization could be accomplished simply by enrolling patients with the understanding that the study is to be a long-term one. Early dropouts from tricyclic treatment can probably be reduced by a slower dosage buildup. Maintaining placebo groups over long periods of time is probably not feasible. If the dropout rate cannot be reduced, it can at least be used as an outcome measure, with a clearer determination of the reasons for dropout than was done in the present study. The pros and cons of endpoint versus completer analyses for the statistical handling of dropouts has been discussed elsewhere (Cross-National Collaborative Panic Study Second Phase Investigators, 1992). In addition to the traditional endpoint and completer analyses, it might also be helpful for clinicians if further analyses were conducted of data from only those patients who complete the study. This is traditionally not done because the completers are considered not representative of the initially randomized group. However, this is the only way of knowing what actually happens to patients who actually stay with a particular treatment. Such information is not accurately reflected by the present practice of reporting and analyzing mean scores of a group which diminishes in size at each time point, since this provides no exact measure of the initial scores of those patients who completed the study, and are consequently the only ones whose scores are available at the final time point.

\section{Conclusions}

These results suggest that in cases where long-term treatment is needed, either alprazolam or imipramine may be a viable option. On average, as far as efficacy, patient acceptance, and safety while on medication are concerned, there appears to be little basis for choosing either of these two drugs over the other. Such faint and uncertain advantages as there may be, appear slightly to favor imipramine. Ease of discontinuation also favors imipramine, though discontinuation of alprazolam is also quite manageable if one employs patience and understands the principles involved (Abelson \& Curtis, 1993). Of great potential importance is the recent report (Mavissakalian \& Perel, 1992) that after 6 months on imipramine, relapse can be prevented for up to an additional year by maintenance on half the original dose. Whether the same is possible with benzodiazepines remains to be determined. Since the advantages of one agent are mostly at the beginning of treatment, and the advantages of the other seem to be mainly over the longer term, one naturally thinks of either starting both together and later tapering away the alprazolam, or of starting with alprazolam, adding imipramine later and then tapering the alprazolam. This does seem to work in the hands of some clinicians, though the time required for tapering the alprazolam may be 
longer than anticipated. As in all clinical decisions, factors specific to individual patients may outweigh other considerations. For example tricyclics must be avoided in patients with heart block and may be problematic in elderly patients or in patients on other drugs with anticholinergic side effects. Benzodiazepines may be problematic in patients with histories of alcohol or drug abuse. The dose of alprazolam (average about $6.5 \mathrm{mg} /$ day, maximum $10 \mathrm{mg} /$ day) was higher than the maximum of $4 \mathrm{mg}$ /day currently recommended in the United States, but similar to those required in the acute studies preceding this one (Ballenger, 1990; Ballenger et al., 1988; Cross-National Collaborative Panic Study Second Phase Investigators, 1992). The higher doses are well tolerated when needed for symptom control. The currently recommended doses in the United States appear to be too low for optimal effectiveness. A number of other countries do recommend doses of up to $10 \mathrm{mg} /$ day.

A question of major interest is how long drug therapy should be continued. This question cannot be answered as clearly as it can be for depression, because the necessary work has not yet been done. About as much as one can say at present is that the relapse rate appears to be high after acute drug trials and the illness appears to be chronic, perhaps lifelong, in some patients. A reasonable approach would seem to be to treat with drugs until both patient and therapist are comfortable that stable symptom control has been achieved. Then with the patient's agreement, a search can be initiated for the minimum dose which controls symptoms. In the case of benzodiazepines, patient and therapist must both understand that a temporary period of rebound anxiety may follow each dose reduction. Partial relapses may be treatable with cognitive/behavioral methods. Using an approach similar to this, Abelson and Curtis (1993) found 78\% of their alprazolam treated patients could discontinue benzodiazepines and that only $36 \%$ of those relapsed. Some 1 to 2 years after enrollment, $61 \%$ of those followed up were medication free. However, the taper period occasionally lasted up to 18 months.

Acknowledgments-The authors thank Philip Lavori, Ph.D. for statistical consultation, and David Shera for assistance in the performance of statistical analyses. Professor Jean Wilmotte, M.D. was project director of the Belgian Study site.

\section{References}

Abelson, J. L., \& Curtis, G. C. (1993). Discontinuation of alprazolam after successful treatment of panic disorder: A naturalistic follow-up study. Journal of Anxiety Disorders, 7, 107-117.

Aronson, T. (1989). A naturalistic study of imipramine in panic disorder and agoraphobia. American Journal of Psychiatry, 144, 1014-1019.

Ballenger, J. (1986). Pharmacotherapy of the panic disorders. Journal of Clinical Psychiatry, 47 (Suppl. 6), 2732.

Ballenger, J. (1990). Efficacy of benzodizepines in panic disorder and agoraphobia. Journal of Psychiatric Research, 24 (Suppl. 2), 15-25.

Ballenger, J., Burrows, G., DuPont, R., Jr, Lesser, I., Nnyes, R., Jr, Pecknold, J., Rifkin, A., \& Swinson, R. (1988). Alprazolam in panic disorder and agoraphobia: results from a multicenter trial: I. Efficacy in shortterm treatment. Archives of General Psychiatry, 45, 413-422.

Burrows, G. (1990). Managing long term therapy for panic disorder. Journal of Clinical Psychiatry, 51(11) (Suppl.), 9-12.

Cassano, G. (1990). Compliance and safety in second phase of Cross-National collaborative study. Journal of Psychiatric Research, 24 (Suppl. 1), 43.

Cross-National Collaborative Panic Study Second Phase Investigators (1992). Drug treatment of panic disorder: Comparative efficacy of alprazolam, imipramine and placebo. British Journal of Psychiatry, 160, 191-202. 
Katschnig, H. (1990). Long-term follow-up of treated panic disorder. Journal of Psychiatric Research, 24 (Suppl. 1), 29-30.

Klerman, G. (1988). Overview of the cross-national collaborative panic study. Archives of General Psychiatry, $\mathbf{4 5}$, $407-412$.

Klerman, G., Coleman, J., \& Purpura, R. (1986). The design and conduct of the Upjohn Cross-National Collaborative Panic Study. Psychopharmacology Bulletin, 22, 5975.

Levine, J., \& Schooler, N. R. (1986). SAFETEE: A technique for the systematic assessment of side effects in clinical trials. Psychopharmacology Bulletin, 22, 343-381.

Liebowitz, M. (1985). Imipramine in the treatment of panic disorder and its complications. Psychiatric Clinics of North America, 3, 2747.

Mavissakalian, M., \& Perel, J. M. (1992). Clinical experiments in maintenance and discontinuation of imipramine therapy in panic disorder with agoraphobia. Archives of General Psychiatry, 49, 318323.

Nagy, L., Krystal, J., Woods, S., \& Charney, D. (1989). Clinical and medication outcome after short-term alprazolam and behavioral group treatment in panic disorder. Archives of General Psychiatry, 46, 993-999.

Noyes, R., Jr, DuPont, R., Pecknold, J., Rifkin, A., Rubin, R., Swinson, R., Ballenger, J., \& Burrows, G. (1988). Alprazolam in panic disorder and agoraphobia: Results from a multicenter trial: II. Patient acceptance, side effects, and safety. Archives of General Psychiatry, 45, 423.436.

Noyes, R., Jr, Garvey, M., Cook, B., \& Samuelson, R. (1989). Problems with tricyclic antidepressant use in patients with panic disorder or agoraphobia: Results of a naturalistic follow-up study. Journal of Clinical Psychiatry, 50, 163-169.

Noyes, R., Jr, Reich, J., Christiansen, J., Suelzer, M., Pfohl, B., \& Coryell, W. (1990). Outcome of panic disorder. Archives of General Psychiatry, 47, 809-818.

Pecknold, J., Swinson, R., Kuch, K., \& Lewis, C. (1988). Alprazolam in panic disorder and agoraphobia: Results from a multicenter trial. III. Discontinuation effects. Archives of General Psychiatry, 45, 129-136.

Pollack, M. (1990). Long-term management of panic disorder. Journal of Clinical Psychiatry, 51(5) (Suppl.), 11 13.

Pollack, M., Otto, M., Rosenbaum, J., Sachs, G., O'Neil, C., Asher, R., \& Meltzer-Brody, S. (1990). Longitudinal course of panic disorder: Findings from the Massachusetts General Hospital Naturalistic Study. Journal of Clinical Psychiatry 51(12) (Suppl. A), 12-16.

Pollack, M., Tesar, G., Rosenbaum, J., \& Speir, G. (1986). Clonazepam in the treatment of panic disorder and agoraphobia: A one-year follow-up. Journal of Clinical Psychopharmacology, 6, 302-304.

Rosenberg. R. (1990). Results of Phase II discontinuation study. Journal of Psychiatric Research, 24 (Suppl. 1), 82. 